The Egyptian Journal of Hospital Medicine (October 2020) Vol. 81 (2), Page 1379-1388

\title{
Role of Metformin in Combination with Neoadjuvant Chemotherapy in Treatment of Breast Cancer
}

\author{
Ahmed Said Ahmed Said ${ }^{* 1}$, Hussein Ali Mustafa abd Al-Mutteleb², Mohammed Ali Morsy Abdeen ${ }^{3}$, \\ Mohammed Abd Al Rahman Hassan ${ }^{3}$, Soha Abdelrazek Abbas Ahmed ${ }^{1}$ \\ Departments of ${ }^{1}$ Clinical Oncology \& Nuclear Medicine and \\ ${ }^{2}$ General Surgery, Faculty of Medicine- Aswan University \\ Department of ${ }^{3}$ Clinical Oncology \& Nuclear Medicine, Faculty of Medicine- Cairo University \\ *Corresponding author: Ahmed Said Ahmed Said, Mobile: (+20) 01125408705, E-Mail: ahmedsaidorman@ yahoo.com
}

\begin{abstract}
Backgound: Globally, breast cancer is the most frequently diagnosed malignancy and the leading cause of cancer death in women. Improvements in chemotherapy, surgery, lymph node evaluation and hormone receptor blocking therapy have successfully doubled the survival of breast cancer patients.

Objective: To determine whether metformin use was associated with improvement in pathologic complete response (PCR) rate in patients with breast cancer receiving neoadjuvant chemotherapy.

Patients and Methods: This prospective study was conducted at the Clinical Oncology Department, Aswan University and Upper Egypt Hospitals in the period between 1/7/2016 and 1/9/2019. This study included 50 patients divided into 2 groups, test group (metformin group) and standard group (non-metformin group). Histopathology was confirmed by tissue core biopsy.

Results: All patients in the two groups in our study achieved either pathological complete response or partial response. No patients developed disease progression or were still stable disease. Among the patients in test group (metformin group), 15 patients $(\mathbf{6 0 \%})$ achieved pathological complete response (PCR) while 10 (40\%) patients did not achieve PCR. However, among the patients in standerd group (non-metformin group) 9 patients (36\%) achieved pathological complete response (PCR) while $16(64 \%)$ patients did not achieve PCR. There was statistically significant difference between the two groups regarding the response with better PCR in metformin group ( $\mathrm{p}$ value is 0.089 ).
\end{abstract}

Conclusion: The addition of metformin to neoadjuvant chemotherapy has a significant impact on pathological complete response (PCR) in female patients with advanced breast cancer.

Keywords: Metformin, Neoadjuvant Chemotherapy, Breast Cancer

\section{INTRODUCTION}

In general, patients with early-stage breast cancer undergo primary surgery (lumpectomy or mastectomy) to the breast and regional nodes with or without radiation therapy (RT). Over the years, surgery for breast cancer has become less invasive in both the breast and axilla. Surgical techniques have advanced to provide better cosmesis in breast conservation and also in breast reconstruction for woman who require mastectomy ${ }^{(\mathbf{1})}$.

Following definitive local treatment, adjuvant systemic therapy may be offered. Immunohistochemistry (IHC) markers, together with clinicopathological variables such as tumor size, tumor grade, nodal involvement, histologic type and surgical margins, have been widely used for prognosis, prediction and treatment selection ${ }^{(2)}$.

Preoperative systemic therapy (neoadjuvant) is becoming popular nowadays for early-stage or locally advanced breast cancer. Neoadjuvant treatment also allows prompt tumor response evaluation and the residual disease can be a prognostic factor of tumor recurrence and overall survival. In the last decade, pathological response for neoadjuvant chemotherapy, as an indicator for long term clinical benefit in breast cancer, was an evolving chance for more rapid incorporation of new drugs ${ }^{(3)}$.

Metformin, the famous oral hypoglycemic agent, has attracted attention recently for its possible antineoplastic effect ${ }^{(4)}$. A recent convergence of clinical and epidemiologic evidence has linked hyperinsulinemia, insulin resistance and diabetes to poor breast cancer outcomes. This has been coupled with enhanced understanding of molecular effects of metformin and its potential role in malignancy. It has been revealed that metformin may influence cancer cells through indirect (insulin-mediated) effects, or it may directly affect cell proliferation and apoptosis of cancer cells ${ }^{(5)}$. Metformin has shown anticancer properties, including activation of the AMP-activated protein 21 kinase (AMPK) pathway, antioxidant activity, induction of apoptosis and many others. Patients with type 2 diabetes are hyperinsulinemic, and there is evidence that this condition contributes to tumorigenesis ${ }^{(6)}$.

In this scenario, metformin reverses, at least partially, hyperinsulinemia and exhibits antiproliferative properties, which might increase the effect of chemotherapy in a synergy. Preclinical research has demonstrated that metformin can sensitize 
tumor cells to chemotherapy through inhibition of the expression of PI3K/AKT proteins and, consequently, controlling the activity of the mammalian target of rapamycin (mTOR). The combination of metformin with chemotherapy appears to inhibit mTOR activity, inducing cell cycle arrest ${ }^{(7)}$.

Generally, metformin has anti-tumor effects through two mechanisms: 1) a direct mechanism (independent of insulin); and 2) an indirect mechanism (insulin-dependent). In the indirect mechanism, metformin activates AMPK, which results in prevention of the transcription of the gene responsible for glycogenesis in liver cells. In this process, glycogenosis decreases and, as a result, glucose uptake in muscle cells increases. Glucose uptake in the muscle cells leads to a decrease in blood glucose levels and subsequently insulin levels. Since high levels of insulin in the blood, due to the high number of insulin receptors in the cancer cells, have mitogenic effects and can cause tumor growth and proliferation, reducing insulin levels in the blood reduces the likelihood of malignity and prevents cancer cell proliferation ${ }^{(8)}$.

\section{AIM OF THE WORK}

This prospective study aimed to determine whether metformin use was associated with improvement in pathologic complete response (PCR) rate in patients with breast cancer receiving neoadjuvant chemotherapy.

\section{PATIENTS AND METHODS}

Our prospective study was conducted at the Clinical Oncology Department, Aswan University and Upper Egypt Hospitals in the period between 1/7/2016 and 1/9/2019. 50 female patients with breast cancer who were eligible to receive neoadjuvant chemotherapy were included. We divided female patients with breast cancer into 2 groups: Twenty-five patients received neoadjuvant chemotherapy AC-Taxol + metformin (test group) and Twenty-five patients received neoadjuvant chemotherapy AC-Taxol (standard group) without metformin.

Inclusion Criteria: Female patients with histological and radiological proof of non-metastatic breast cancer. Age: from 18 to 70 years old. Female patients with clinical T2 Breast cancer or more and/or clinical positive LN. Performance status: 0-2 WHO. Patients with adequate left ventricular ejection fraction $(\geq 50 \%)$ and normal hematological, renal and hepatic functions. Exclusion Criteria: Bilateral tumour. Patients submitted to excisional biopsy from breast mass. Patients who are pregnant or lactating. Patints with double malignancy. Presence of metastatic disease at diagnosis. Patients refuse to be conducted in the study. Patients that are developing severe complications from the use of treatment. Diabetic patients already on metformin therapy. Withdrawal of concent at any time during the study.

\section{Pretreatment evaluation:}

1. Medical history and physical examination.
2. Sono-mamography or MRI breast if needed.

3. CT chest, abdomen and pelvis.

4. Bone scan.

5. Echocardiography and left ventricular ejection fraction.

6. Fasting and postprandial blood sugar.

7. Baseline laboratory investigation (CBC, LFT \& KFT).

8. Assesment of ER, PR and Her-2 neu status on pathology specimen.

\section{During treatment evaluation:}

1. Physical examination before each cycle.

2. CBC, LFT and KFT before each cycle.

3. Fasting and postprandial blood sugar.

\section{Drug therapy:}

1. Four cycles AC followed by 12 weeks paclitaxel single agent $80 \mathrm{mg} / \mathrm{m}^{2}$ plus metformin $500 \mathrm{mg}$ twice/day until the time of surgery versus four cycles AC followed by 12 weeks paclitaxel single agent $80 \mathrm{mg} / \mathrm{m}^{2}$ without metformin.

2. Trastazumab was given for the patients with Her2/neu positive by IHC or FISH if financial support was found.

3. Adjuvant hormonal treatment in hormonal receptors-positive patients.

\section{Surgery:}

Two to three weeks after full course of chemotherapy.

Patients were evaluated after the end of treatment according to RECIST criteria (Response Evaluation Criteria in Solid Tumors):

\section{A. Evaluation of target lesions: (Breast and axillary lymph nodes):}

1. Complete Response (CR): Disappearance of all target lesions and reduction in the short axis measurement of all pathologic lymph nodes to $<10$ $\mathrm{mm}$.

2. Partial Response (PR): $\geq 30 \%$ decrease in the sum of the longest diameter of the target lesions compared to baseline.

3. Progressive Disease (PD): $\geq 20 \%$ increase of at least $5 \mathrm{~mm}$ in the sum of the longest diameter of the target lesions compared to the smallest sum of the longest diameter recorded $\mathbf{O R}$ The appearance of new lesions, including those detected by FDGPET.

4. Stable Disease (SD):

5. Neither sufficient shrinkage to qualify for PR nor sufficient increase to qualify for PD ${ }^{(9)}$.

6. Pathological complete response (PCR): The core of definition of PCR is achieving no residual histological evidence of tumor (Breast and axillary lymph nodes) after chemotherapy at the time of surgery ${ }^{(\mathbf{1 0})}$. 


\section{B. Evaluation of non target lesions:}

1. Pathological Complete response (PCR): Disappearance of all non-target lesions and normalization of tumor marker levels

2. Incomplete Response (IR) or Stationary disease (SD): Persistence of 1 or more non-target lesions and/or the maintenance of tumor marker levels above normal limits.

3. Progressive Disease (PD): The appearance of 1 or more new lesions or unequivocal progression. If patient has measurable disease, an increase in the overall level or substantial worsening in non-target lesions, such that tumor burden has increased, even if there is $\mathrm{SD}$ or PR in target lesions. If not measurable disease, an increase in the overall tumor burden comparable in magnitude with the increase that would be required to declare PD in measurable disease ${ }^{(9)}$.

Ethical approval and written informed consent:

An approval of the study was obtained from Aswan University Academic and Ethical Committee. Every patient signed an informed written consent for acceptance of the operation.

\section{Statistical analysis}

Recorded data were analyzed using the statistical package for social sciences, version 20.0 (SPSS Inc., Chicago, Illinois, USA). Quantitative data were expressed as mean \pm standard deviation (SD). Qualitative data were expressed as frequency and percentage.

\section{The following tests were done:}

- Independent-samples t-test of significance was used when comparing between two means.
- Chi-square $\left(\mathrm{x}^{2}\right)$ test of significance was used in order to compare proportions between two qualitative parameters.

- The confidence interval was set to $95 \%$ and the margin of error accepted was set to 5\%. The pvalue significance was considered as the following:

- Probability (P-value)

- $\quad P$-value $\leq 0.05$ was considered significant.

- P-value < 0.001 was considered as highly significant.

- P-value > 0.05 was considered insignificant.

\section{RESULTS}

\section{Epidemiological characterestics (Table 1):}

The median age in test group was 49 years, ranged from (31-65 years) while the median age in standard group was 45 years, ranged from (24-65 years). In test group, 15 patients (60\%) were premenopausal while 10 patients $(40 \%)$ were postmenopausal. In standard group, 18 patients (72\%) were premenopausal while 7 patients were postmenopausal (28\%). There was no statistically significant difference between the two groups regarding age ( $\mathrm{p}$ value 0.31 ) and menopausal status ( $\mathrm{p}$ value 0.37 ). There was no statistically significant difference between the two groups regarding weight ( $\mathrm{p}$ value 0.190 ), height ( $\mathrm{p}$ value 0.540 ) and $\mathrm{BMI}$ ( $\mathrm{p}$ value 0.271 ). There was no statistically significant difference between the two groups regarding diabetes mellitus ( $\mathrm{p}$ value 0.289 ). There was no statistically significant difference between the two groups regarding hypertension (HTN) (p value 0.463 ).

Table (1): Epidemiological characterestics and comorbidities

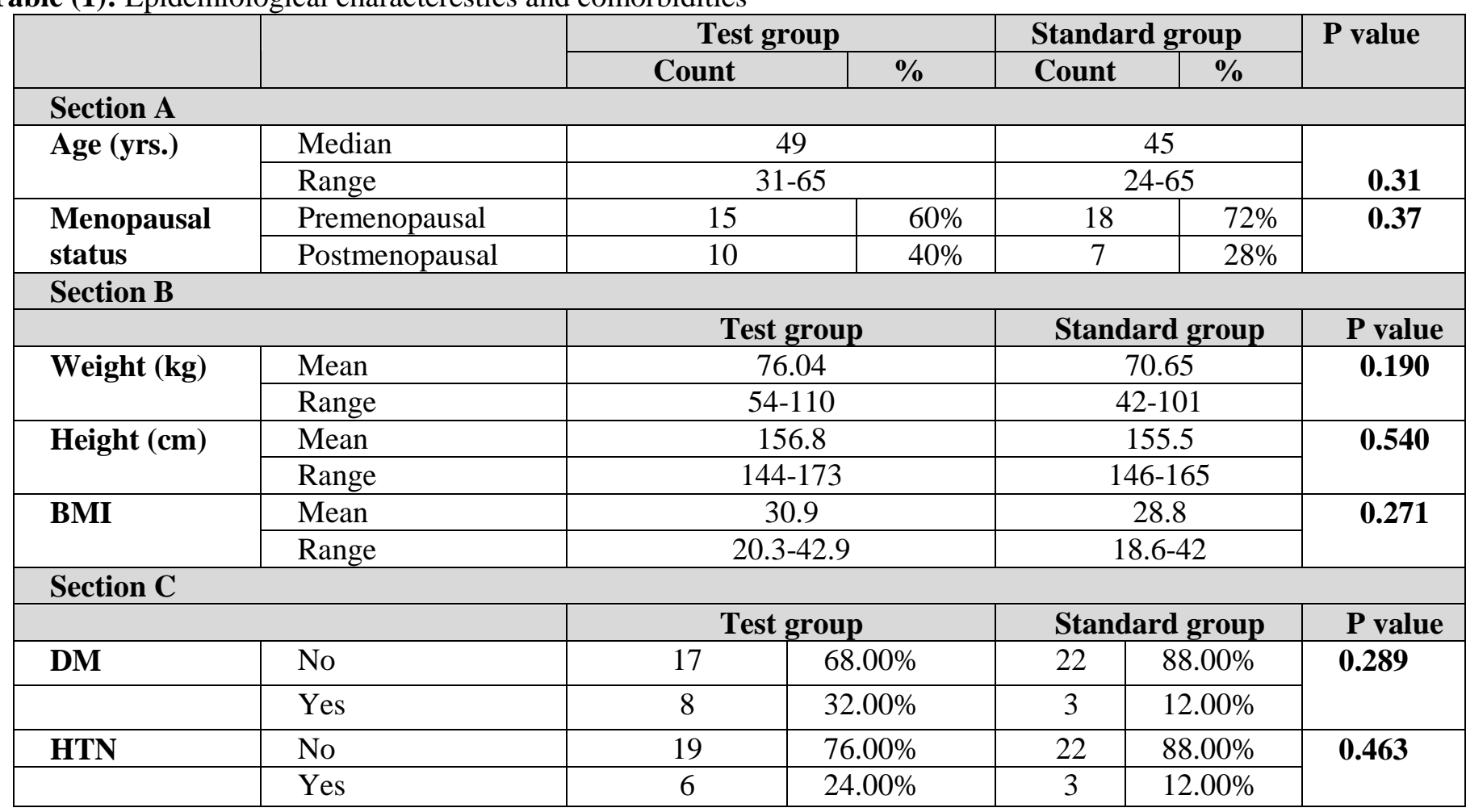




\section{Pathological characterestics (Table 2):}

All patients in the 2 groups were submitted to tissue core biopsy from suspicious malignant lesion and were evaluated according to pathological subtypes, hormonal receptor status and biological stratification. All patients in both groups were proved to have invasive breast cancer by tissue core biopsy. Among the patients in 2 groupes, pathological subtypes detected was as follows: Invasive duct carcinoma (IDCa), invasive lobular carcinoma (ILCa), mixed invasive duct carcinoma (mixed IDCa) and mixed lobular carcinoma (mixed ILCa). There was no statistically significant difference between the two groups regarding pathological subtypes ( $p$ value 0.7 ). There was no statistically significant difference between the two groups regarding ER ( $\mathrm{p}$ value 0.77 ), PR ( $\mathrm{p}$ value 0.556 ) or HER-2/neu ( $p$ value 0.569 ). There was no statistically significant difference between the two groups regarding luminal subtypes ( $\mathrm{p}$ value 0.535 )

Table (2): Pathological characterestics

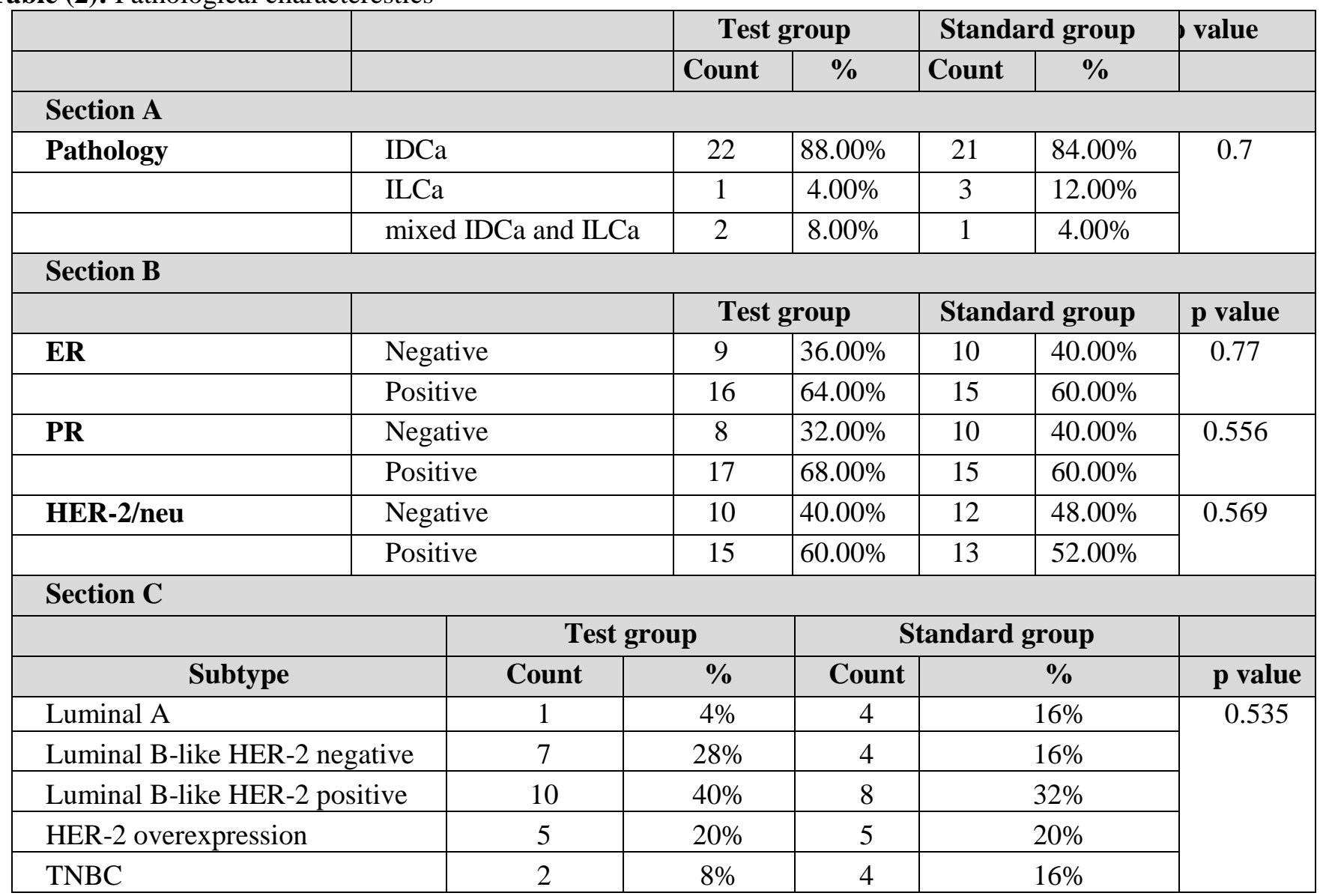

\section{Surgical intervention post neoadjuvant} chemotherapy (Table 3):

All patients in the 2 groups were submitted to surgery after end of neoadjuvant chemotherapy. All patients were submitted to either modified radical mastectomy (MRM) or conservative breast surgery (CBS).
Among the patients in test group, 19 patients (76\%) were submitted to MRM while 6 patients (24\%) were submitted to CBS. Among the patients in standerd group, 23 patients (92\%) were submitted to MRM while 2 patients $(8 \%)$ were submitted to CBS.

There was no statistically significant difference between the two groups regarding types of surgery ( $\mathrm{p}$ value 0.247 ).

Table (3): Surgical intervention

\begin{tabular}{|l|l|c|c|c|c|c|}
\hline \multirow{2}{*}{} & & \multicolumn{2}{|c|}{ Metformin } & \multicolumn{2}{c|}{ No metformin } & \multirow{2}{*}{} \\
\cline { 3 - 7 } & & Count & \% & Count & \% & p value \\
\hline \multirow{2}{*}{ Surgery } & MRM & 19 & $76.00 \%$ & 23 & $92.00 \%$ & 0.247 \\
\cline { 2 - 6 } & CBS & 6 & $24.00 \%$ & 2 & $8.00 \%$ & \\
\hline
\end{tabular}




\section{Response (Table 4):}

\section{1- Clinical response:}

All patients in the 2 groups were evaluated clinically after the end of chemotherapy. No patients developed clinical progressive disease or stable disease.

There was no statistically significant difference between the two groups regarding $\mathrm{T}$ staging postneoadjuvant chemotherapy ( $\mathrm{p}$ value 0.393 ). There was no statistically significant difference between the two groups regarding $\mathrm{N}$ staging post neoadjuvant chemotherapy ( $\mathrm{p}$ value 0.343 ).

\section{2- Radiological response:}

All patients in the 2 groups were evaluated radiologically by breast ultrasound and mammography after the end of chemotherapy to detect any residual suspicious breast mass or residual suspicious lymph nodes. All patients achieved either complete remission or regressive disease. No patients developed radiological progressive disease or stable disease.

There was no statistically significant difference between the two groups regarding residual breast mass postneoadjuvant chemotherapy ( $\mathrm{p}$ value 0.156 ). There was no statistically significant difference regarding radiological residual LNs postneoadjuvant chemotherapy ( $\mathrm{p}$ value 0.417 ).

\section{3- Pathological response:}

All patients in the 2 groups were evaluated pathologicaly after surgical intervention post neoadjuvant chemotherapy whether the patients achieved PCR or not. All patients in 2 group achieved either pathological complete response or partial response.

There was nearby statistically significant difference between the two groups regarding the response with better PCR in metformin group ( $\mathrm{p}$ value is 0.089 ).

Table (4): Clinical, radiological and pathological responses

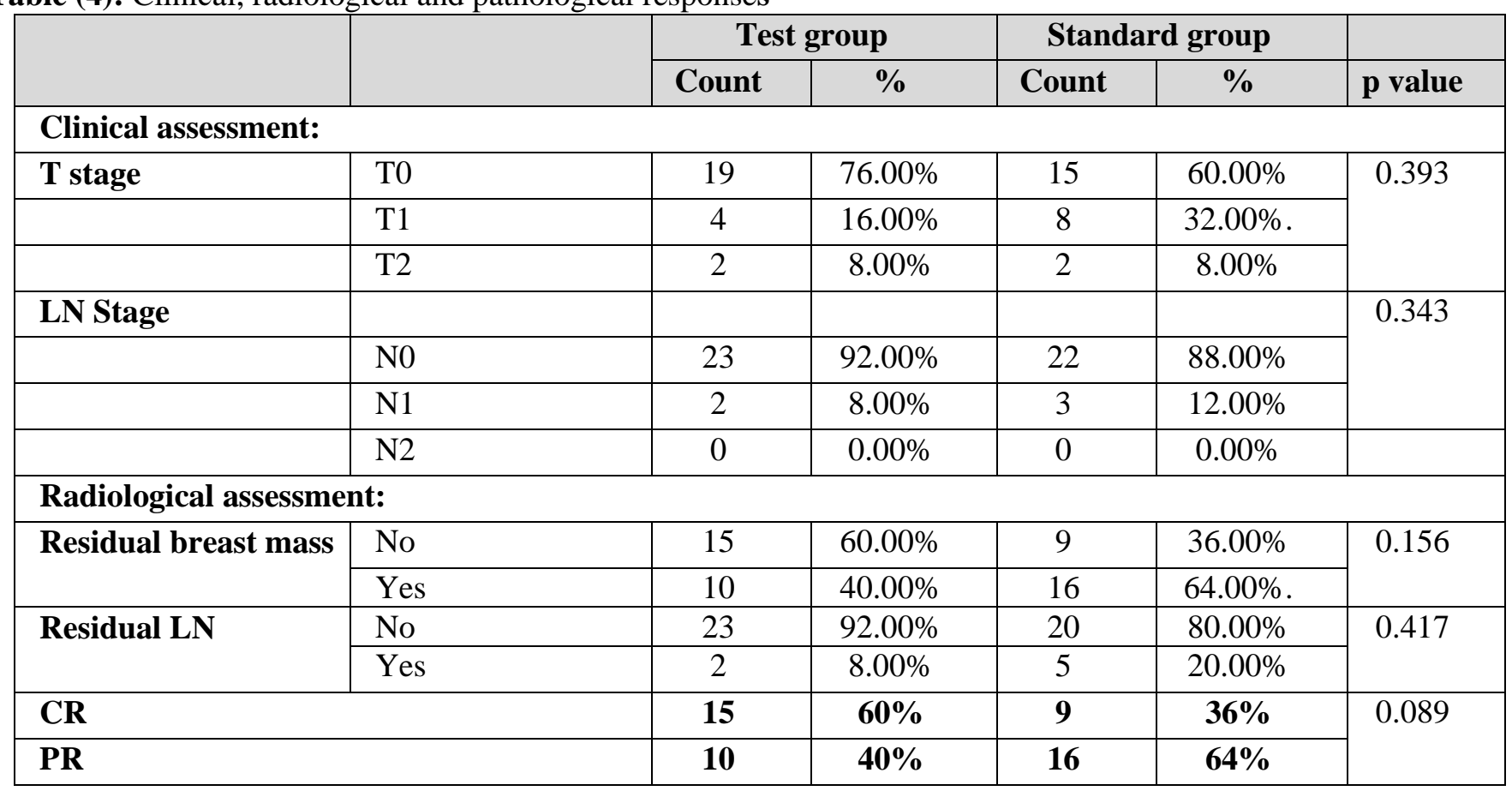

\section{Correlation between PCR and different criteria (Table 5):}

The correlation between PCR in the 2 groups and other variables including clinical and pathological charecterestics. It showed significant correlation between PCR in the 2 groups concerning ER positivity and HER-2/neu positivity. While there was no significant correlation between PCR in the 2 groups and other variable charecterstics regarding age, menopausal status, site of the disease, comorbidities, preoperative $\mathrm{T}$ stage, preoperative $\mathrm{N}$ stage, $\mathrm{PR}$, preoperative staging, pathology or luminal subtypes. 
Table (5): Correlation between PCR and different criteria

\begin{tabular}{|c|c|c|c|c|}
\hline Criteria & & $\begin{array}{c}\text { PCR in } \\
\text { Metformin }\end{array}$ & $\begin{array}{l}\text { PCR in Non } \\
\text { metformin }\end{array}$ & $\begin{array}{c}\text { Correlation } \\
\text { P value }\end{array}$ \\
\hline \multirow{2}{*}{ Age } & $<50$ & $40 \%$ & $24 \%$ & \multirow{2}{*}{ - } \\
\hline & $>50$ & $20 \%$ & $12 \%$ & \\
\hline \multirow[t]{2}{*}{ Menopause } & Pre-menopause & $44 \%$ & $24 \%$ & \multirow[b]{2}{*}{0.492} \\
\hline & Post-menopause & $16 \%$ & $12 \%$ & \\
\hline \multirow[t]{6}{*}{ Site of disease } & LOQ & $4 \%$ & $12 \%$ & \multirow{6}{*}{0.575} \\
\hline & Retroareolr & $28 \%$ & $4 \%$ & \\
\hline & UIQ & $0 \%$ & $4 \%$ & \\
\hline & UOQ & $20 \%$ & $8 \%$ & \\
\hline & LIQ & $4 \%$ & $4 \%$ & \\
\hline & Axilla & $4 \%$ & $4 \%$ & \\
\hline \multirow[t]{2}{*}{ Clinical staging } & IIIA & $48 \%$ & $32 \%$ & \multirow[b]{2}{*}{0.263} \\
\hline & IIIB & $12 \%$ & $4 \%$ & \\
\hline \multirow[t]{3}{*}{ Preoperative T } & $\mathrm{T} 2$ & $4 \%$ & $4 \%$ & \multirow{3}{*}{0.202} \\
\hline & T3 & $44 \%$ & $28 \%$ & \\
\hline & $\mathrm{T} 4$ & $12 \%$ & $4 \%$ & \\
\hline \multirow[t]{2}{*}{ Preoperative $\mathbf{N}$} & N1 & $28 \%$ & $20 \%$ & \multirow{2}{*}{0.404} \\
\hline & $\mathrm{N} 2$ & $32 \%$ & $16 \%$ & \\
\hline \multirow[t]{3}{*}{ Pathology } & IDCa & $52 \%$ & $32 \%$ & \multirow{3}{*}{0.834} \\
\hline & ILCa & $4 \%$ & $0 \%$ & \\
\hline & Mixed & $4 \%$ & $4 \%$ & \\
\hline \multirow[t]{3}{*}{ Comorbidities } & DM & $12 \%$ & $8 \%$ & \multirow{3}{*}{0.409} \\
\hline & HTN & $16 \%$ & $4 \%$ & \\
\hline & No & $40 \%$ & $28 \%$ & \\
\hline \multirow[t]{2}{*}{ ER } & $+\mathrm{ve}$ & $32 \%$ & $16 \%$ & \multirow[b]{2}{*}{0.004} \\
\hline & -ve & $28 \%$ & $20 \%$ & \\
\hline \multirow[t]{2}{*}{ PR } & $+\mathrm{ve}$ & $32 \%$ & $20 \%$ & \multirow[b]{2}{*}{0.835} \\
\hline & -ve & $28 \%$ & $16 \%$ & \\
\hline \multirow[t]{2}{*}{ HER-2 } & $+\mathrm{ve}$ & $52 \%$ & $28 \%$ & \multirow{2}{*}{0.004} \\
\hline & -ve & $8 \%$ & $8 \%$ & \\
\hline \multirow[t]{5}{*}{ Luminal } & $\mathrm{A}$ & $0 \%$ & $0 \%$ & \multirow{5}{*}{0.295} \\
\hline & B-HER-2 -ve & $8 \%$ & $0 \%$ & \\
\hline & B-HER-2 +ve & $32 \%$ & $20 \%$ & \\
\hline & $\begin{array}{l}\text { HER-2 } \\
\text { overexpression }\end{array}$ & $20 \%$ & $8 \%$ & \\
\hline & TNBC & $0 \%$ & $8 \%$ & \\
\hline
\end{tabular}

\section{Regarding Adjuvant Hormonal Treatment (Table} 6):

In test group: 18 patients (72\%) received adjuvant hormonal treatment while 7 patients $(28 \%)$ not received adjuvant hormonal treatment.

In standard group: 15 patients $(60 \%)$ received adjuvant hormonal treatment while 10 patients $(40 \%)$ did not receive adjuvant hormonal treatment.

Among the patients that received adjuvant hormonal treatment:

In test group, 7 patients (28\%) received aromatase inhibitors (AI) while 11 patients (44\%) received tamoxifen.
In standard group, 4 patients (16\%) received aromatase inhibitors (AI) while 11 patients (44\%) received tamoxifen.

\section{Regarding Targeted therapy (herceptin):}

In test group, 15 patients $(60 \%)$ received neoadjuvant and adjuvant herceptin (complete 1 year) treatment while 10 patients (40\%) did not receive herceptin either neoadjuvant or in adjuvant setting.

In standard group, 13 patients $(52 \%)$ received neoadjuvant and adjuvant herceptin (complete 1 year) treatment while 12 patients $(48 \%)$ did not receive herceptin either neoadjuvant or in adjuvant setting. 
Table (6): Adjuvant hormonal and target therapy

\begin{tabular}{|l|l|c|c|c|c|c|}
\hline & & \multicolumn{2}{|c|}{ Metformin } & \multicolumn{2}{c|}{ No metformin } & \\
\hline & & Count & \% & Count & \% & p value \\
\hline \multirow{2}{*}{$\begin{array}{l}\text { Adjuvant Hormonal } \\
\text { Treatment }\end{array}$} & No & 7 & $28.00 \%$ & 10 & $40.00 \%$ & 0.551 \\
\cline { 2 - 6 } $\begin{array}{l}\text { Adjuvant Hormonal } \\
\text { Treatment }\end{array}$ & Yes & 18 & $72.00 \%$ & 15 & $60.00 \%$ & \\
\cline { 2 - 6 } & Tamoxifen & 7 & $28.00 \%$ & 4 & $16.00 \%$ & 0.712 \\
\cline { 2 - 6 } Adjuvant Herceptin & No & 11 & $44.00 \%$ & 11 & $44.00 \%$ & \\
\cline { 2 - 6 } & Yes & 15 & $60.00 \%$ & 12 & $48.00 \%$ & \multirow{2}{*}{0.569} \\
\hline
\end{tabular}

\section{Survival analysis (Table 7):}

The mean survival time for cases fulfiled in this study was $37.393 \pm 0.905$. Kaplan-Meier survival analysis showed that survival time was not significantly shorter among women treated with AC/ Taxol combined with metformin (mean survival time was 37 \pm 0.979 ) than those treated without metformin (mean survival time was $36.85 \pm 1.47$ ).

Table (7): Survival analysis

\begin{tabular}{|c|c|c|c|c|}
\hline Mean survival time (months) & Standard error & \multicolumn{3}{|c|}{$95 \% \mathrm{CI}$} \\
\hline \multirow[t]{3}{*}{37.393} & 0.905 & \multicolumn{3}{|c|}{$35.619-39.167$} \\
\hline & \multirow{2}{*}{$\begin{array}{l}\text { Mean survival time } \\
\text { by months }\end{array}$} & \multicolumn{3}{|c|}{ P-value } \\
\hline & & $\begin{array}{c}\text { Log rank } \\
\text { test }\end{array}$ & $\begin{array}{c}\text { Breslow } \\
\text { test }\end{array}$ & $\begin{array}{c}\text { Tarone-ware } \\
\text { test }\end{array}$ \\
\hline \multicolumn{2}{|l|}{ Treatment: } & \multirow{3}{*}{0.566} & \multirow{3}{*}{0.622} & \multirow{3}{*}{0.595} \\
\hline AC/Taxol & $37 \pm 0.979$ & & & \\
\hline AC/Taxol + Metformin & $36.85 \pm 1.47$ & & & \\
\hline
\end{tabular}

\section{DISCUSSION}

The role of metformin with neoadjuvant chemotherapy in breast cancer had been studied frequently. In a retrospective study that involved 2, 529 patients who received neoadjuvant chemotherapy for early-stage breast cancer between 1990 and $2007^{(11)}$, patients were compared by groups: 68 diabetic patients taking metformin, 87 diabetic patients not taking metformin, and 2, 374 non-diabetic patients who did not receive metformin. Pathological complete response (PCR) rate was $24 \%$ in the metformin group, $8.0 \%$ in the non-metformin group, and $16 \%$ in the non-diabetic group, i.e. diabetic patients with breast cancer who have received metformin and neoadjuvant chemotherapy had a significantly higher PCR rate than did diabetics not receiving metformin.

In another cross sectional study that involved 53 patients who received neoadjuvant chemotherapy for early-stage or locally advanced breast cancer receiving neoadjuvant systemic treatment from January 2007 to December $2015^{(\mathbf{1 2})}$, patients were divided into two groups: 14 received metformin with systemic therapy, and 39 had systemic therapy alone. The PCR rate in the metformin group was $64.3 \%$ compared to $23.1 \%$ in the systemic therapy-alone group.

In another a phase 2 trial of neoadjuvant metformin in combination with trastuzumab and chemotherapy, Martin-Castillo et al. ${ }^{(13)}$ studied 58 patients who received neoadjuvant chemotherapy in women with early HER2- positive breast cancer between June 2012 and March 2016. The patients were divided into two groups: 29 received metformin with systemic therapy plus trastuzumab, and 29 had systemic therapy plus trastuzumab without metformin. PCR rate was numerically higher in the metformincontaining arm A $65.5 \%$ (19 of 29 patients) than in arm B $58.6 \%$ (17 of 29 patients).

In our study, 50 female patients with breast cancer received neoadjuvant chemotherapy were included. We divide female patients with breast cancer into 2 groups: 25 patients received neoadjuvant chemotherapy ACTaxol+ metformin (test group) and 25 patients received neoadjuvant chemotherapy AC-Taxol (standerd group) without metformin. The median age in the test group was 49 years old (ranging from 31-65 years) while the median age in the standerd group was 45 years old (ranging from 24-65 years).

The median age in the test group in our current study is younger than metformin groups in Jiralerspong et al. ${ }^{(11)}$ and Van der Laata et al. ${ }^{(14)}$ studies (49 in test group of our current study while 57.5 and 50.3 years respectively in their studies). While, it was older than that in metformin group in MartinCastillo et al. ${ }^{(13)}$ study (47.2 years).

The median age in the standard group was younger than the median age of non-metformin groups 
in Jiralerspong et al. ${ }^{(11)}$, Martin-Castillo et al. ${ }^{(13)}$ and Van der Laata et al. ${ }^{(14)}$ studies (45 in standard group of our current study while $\mathbf{5 7 , 4 9 , 5 3 . 1}$ and 48 years respectively in the other studies).

In our current study, majority of patients were premenopausal $(60 \%$ in test group and $72 \%$ in standard group). This percent is higher than percent of premenopusal patients in Jiralerspong et al. ${ }^{\text {(11) }}$ study (22\% in metformin group and $16 \%$ and $49 \%$ in nonmetformin group). While, in Martin-Castillo et al. ${ }^{(13)}$ study, percent of premenopusal patients was $64 \%$ in metformin group and 58\% in non-metformin group.

The percent of postmenopausal patients $(40 \%$ in test group and $28 \%$ in standard group). This percent is lower than percent of postmenopusal patients in Jiralerspong et al. ${ }^{(11)}$ study (78\% in metformin group and $48 \%$ and $51 \%$ in non-metformin group). While, in Martin-Castillo et al. (13) study percent of postmenopusal patients was (36\% in metformin group and $42 \%$ in non-metformin group).

The mean Body Mass Index (BMI, $\mathrm{kg} / \mathrm{m} 2$ ) in test group was $30.9 \mathrm{~kg} / \mathrm{m}^{2}$ while in standerd group was $28.8 \mathrm{~kg} / \mathrm{m}^{2}$. The mean BMI in the test group in our current study was lower than BMI in two groups in Jiralerspong et al. ${ }^{(11)}$ study. While, it was higher than mean BMI in third group in Jiralerspong et al. (11) study (30.9 in test group of our current study while 33.8, 32.8 and 26.9 respectively). The mean BMI in standard group was lower than BMI in two groups and higher than mean BMI in third group in Jiralerspong et al. (11) (28.8 in standerd group of our current study while 33.8, 32.8 and 26.9 respectively).

Most of the patients in our study were free of comorbidities as only 9 patients in the test group and 4 patients in standerd group had history of comorbidities (D.M. and Hypertension).

Among patients in test group, 7 patients (28\%) were diabetic while $\mathbf{1 8}$ patients $\mathbf{( 7 2} \%$ ) were not diabetic. 6 patients $(\mathbf{2 8 \%})$ were hypertensive while $\mathbf{1 9}$ patients $(\mathbf{7 2 \%})$ were not hypertensive. Among patients in standerd group, 3 patients $(\mathbf{1 2 \%})$ were diabetic while $\mathbf{2 2}$ patients $(\mathbf{8 8 \%})$ were not diabetic. $\mathbf{3}$ patients $\mathbf{( 1 2 \% )}$ ) were hypertensive while $\mathbf{2 2}$ patients $(\mathbf{8 8} \%)$ were not hypertensive.

In our study, all the patients presented by more advanced local disease than the other groups having much higher percent of clinical stage III (100\%) compared to retrospective and cross sectional studies $(44 \%, 43 \%$ and $40 \%$ were stage III in retrospective study while $50 \%$ and $56 \%$ in cross sectional study). No patients in our current study presented by stage I or stage II disease but in the other studies, percent of stage I disease at presentation was $0 \%, 1 \%$ and $5 \%$ in the Jiralerspong et al. ${ }^{(11)}$ study and $0 \%$ in Van der Laata et al. ${ }^{(14)}$ study. While, percent of stage II disease at presentation was $56 \%, 56 \%$ and $55 \%$ in Jiralerspong et al. $^{\left({ }^{11)}\right.}$ study, and $50 \%$ and $44 \%$ in Van der Laata et al. ${ }^{(14)}$ study.

Esterogen and progesterone receptors (ER, PR) were detected among the patients in our current study. In the test group, ER was positive in 16 patients (64\%) while ER was negative in 9 patients (36\%) and PR was positive in 17 patients $(68 \%)$ while $\mathrm{PR}$ was negative in 8 patients (32\%). In the standerd group, ER and PR were positive in 15 patients $(60 \%)$ while ER, PR were negative in 10 patients $(40 \%)$.

In our study, all patients presented by more advanced local disease than the other groups having much higher percent of clinical stage III (100\%) compared to retrospective and cross sectional studies $(44 \%, 43 \%$ and $40 \%$ were stage III in retrospective study while $50 \%$ and $56 \%$ in cross sectional study). No patients in our current study presented by stage I or stage II disease. But, in the other studies, percent of stage I disease at presentation was $0 \%, 1 \%$ and $5 \%$ in the Jiralerspong et al. ${ }^{(11)}$ study and $0 \%$ in Van der Laata $\boldsymbol{e t}$ al. ${ }^{(14)}$ study. Percent of stage II disease at presentation was 56\%, 56\% and $55 \%$ in the Jiralerspong et al. ${ }^{(11)}$ study and $50 \%$ and $44 \%$ in Van der Laata et al. ${ }^{(14)}$ study.

In addition, Her-2/neu status was detected among the patients in the 2 groups in our study. In test group, HER-2 was positive in 15 patients (60\%) while HER-2 was negative in 10 patients (40\%). In standerd group, HER-2 was positive in 13 patients (52\%) while HER-2 was negative in 12 patients (48\%).

In our study, the percent of negativity of HER$2 /$ neu in the test group was lower than that of the metformin group. In the other studies, it was $69 \%$ in Jiralerspong et al. ${ }^{(11)}$ study and 64\% in Van der Laata et al. (14) study. However, the percent of negativity of HER-2/neu in the standerd group was lower than that of the non-metformin group. In the other studies, it was $70 \%$ in Van der Laata et al. ${ }^{(14)}$ study and $81 \%$ in Jiralerspong et al. $^{(\mathbf{1 1})}$ study.

In our current study, luminal subtypes were detected. Among the patients in test group, 1 patient (4\%) was classified as luminal A, , 7 patient $(28 \%)$ were classified as luminal B-like HER-2 negative, 10 patient $(40 \%)$ were classified as luminal B-like Her-2 positive, 5 patient (20\%) were classified as Her-2 over expression while 2 patient (8\%) were classified as TNBC subtype. However, among the patients in standerd group, 4 patient $(16 \%)$ were classified as Luminal A, 4 patient (16\%) were classified as luminal B-like HER-2 negative, 8 patient (32\%) were classified as Luminal B-like Her-2 positive, 5 patient (20\%) were classified as Her-2 over expression, while 4 patient (16\%) were classified as TNBC subtype.

Although all the patients achieved very good clinical and radiological response with marked reduction of primary lesion, majority of patients 
submitted to modified radical mastectomy (MRM). That could be explained by the knowledge of the benefit of mastectomy among the patients at Upper Egypt as majority of patients think that mastectomy prevent recurrence but the disease may recurs in conservative surgery. Another point, some patients believe that conservative surgery has postoperative complications such as pain and breast edema. So, majority of patients in our current study submitted to modified radical mastectomy (MRM). There was no statistically significant difference between the two groups regarding types of surgery ( $p$ value 0.247 ).

All patients in the two groups in our study achieved either pathological complete response or partial response. No patients developed disease progression or were still stable disease. Among the patients in test group (metformin group), 15 patients (60\%) achieved pathological complete response (PCR) while 10 patients (40\%) did not achieve PCR. However, among the patients in standerd group (nonmetformin group), 9 patients (36\%) achieved pathological complete response (PCR) while 16 patients $(64 \%)$ did not achieve PCR. In our current study, the rate of PCR in the test group was higher than that of the metformin group in Jiralerspong et al. ${ }^{(11)}$ study ( $60 \%$ versus $24 \%$ ) but lower than PCR rate in Van der Laata et al. ${ }^{(14)}$ and Martin-Castillo et al. ${ }^{(13)}$ studies (60\% versus $64.3 \%$ and $65.5 \%$ respectively). However, the rate of PCR in the standard group was higher than that of the non-metformin group in Jiralerspong et al. ${ }^{(11)}$ and Van der Laata et al. ${ }^{(14)}$ studies (36\% versus $8 \%, 16 \%$ and $23.1 \%$ respectively), but lower than PCR rate in Martin-Castillo et al. (13) study (36\% versus $58.6 \%$ ).

In our current study, there was nearby statistically significant difference between the test and standard groups regarding the response with better PCR in metformin group ( $\mathrm{p}$ value is 0.089 ). In Jiralerspong $\boldsymbol{e t}$ al. (11) study, patients were compared by groups: 68 diabetic patients taking metformin, 87 diabetic patients not taking metformin, and 2,374 non-diabetic patients who did not receive metformin. The rate of PCR was $24 \%$ in the metformin group, $8.0 \%$ in the nonmetformin group and $16 \%$ in the nondiabetic group $(\mathrm{P}$ value is 0.02). Pairwise comparisons between the metformin and nonmetformin groups (P value is .007) and the nonmetformin and nondiabetic groups ( $\mathrm{P}$ value is 0.04) were significant. Comparison of the PCR rates between the metformin and nondiabetic groups did not meet significance ( $\mathrm{P}$ value is 0.1 ). This difference could be explaine by difference in number of patients in the two studies (our current study and Jiralerspong $\boldsymbol{e}$ al. (11) study). In our current study, only 50 patients were included divided into 2 groups (test and standard), with equal number in each groups ( 25 patients for each). In Jiralerspong et al. ${ }^{(11)}$ study, patients were compared by groups: 68 diabetic patients taking metformin, 87 diabetic patients not taking metformin, and 2,374 nondiabetic patients who did not receive metformin. Thus, we notice the big difference in total number of population in comparison to our current study and unequality of number of patients in 3 groups in retrospective study.

Another point, although number of patients in test group of our current study was lower than that of metformin group in the retrospective study of Jiralerspong et al. ${ }^{(11)}$ (25 patients versus 68 patients), percent of premenopausal patients was higher in our study than that study ( $60 \%$ versus $22 \%$ ). In addition, nitial clinical stage was more advanced in the test group of our study than that of retrospective study (100\% stage III versus $56 \%$ stage II and $44 \%$ stage III in retrospective study). Percent of HER-2 positivity was higher in test group of our current study $(60 \%$ versus $31 \%)$. PCR rate in our current study was higher than that of retrospective study (60\% versus $24 \%$ ).

In Van der Laata et al. ${ }^{(14)}$ study, that involved 53 patients who received neoadjuvant chemotherapy for early-stage or locally advanced breast cancer, patients were divided into two groups: 14 received metformin with systemic therapy, and 39 had systemic therapy alone. The PCR rate in the metformin group was $64.3 \%$ compared to $23.1 \%$ in the systemic therapy-alone group $(\mathrm{P}=0.008)$. In comparison to our current study, PCR rate in metformin group in Van der Laata et al. (14) study was higher that that of our current study $(64.3 \%$ versus 60\%) with significant difference between metformin and non metformin groups ( $\mathrm{P}$ value was 0.008). However, there was borderline statistically significant difference between the test and standard groups in our study ( $\mathrm{p}$ value was 0.089 ). Although total number of population between our study and Van der Laata et al. ${ }^{(14)}$ study nearly equal (50 patients versus 53 patients in cross sectional study), the difference in PCR rate could be explained by unequality of number of patients in metformin and non-metformin groups (14 patients in metformin group versus 39 patients in nonmetformin group in cross sectional study). There was more advanced initial clinical stage in the 2 groups in our study than that of the cross-sectional study (100\% stage III in whole population in our current study versus $50 \%$ stage II and 50\% stage III in metformin group and $43.6 \%$ stage II and $56.4 \%$ stage III in non-metformin group in the cross sectional study).

In Martin-Castillo et al. ${ }^{(13)}$ study, 58 patients with early HER2-positive breast cancer who received neoadjuvant chemotherapy. They were divided into two groups: 29 received metformin with systemic therapy plus trastuzumab and 29 had systemic therapy plus trastuzumab without metformin. Although number of patients in our current study and Martin-Castillo et

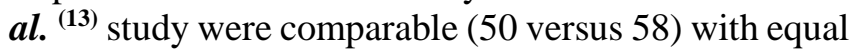


number of patients in each group in the 2 studies. Although, the rate of PCR in Martin-Castillo et al. ${ }^{(13)}$ study was higher than that of our current study, the difference in PCR rate between the 2 groups in MartinCastillo et al. ${ }^{(13)}$ study did not meet statistically significant value (P value was 0.589). However, there was borderline statistically significant difference between the test and standard groups in our current study ( $\mathrm{p}$ value is 0.089). The higher rate of PCR in Martin-Castillo et al. ${ }^{(13)}$ study than that of our current study $(65.5 \%$ versus $60 \%$ in test group and $58.6 \%$ versus $36 \%$ in standard group) could be explained by the use of trastuzumab in all patients. In MartinCastillo et al. ${ }^{(13)}$ study, the whole population were HER2- positive, which play a significant role in achieving PCR. (Buzatto et al. ${ }^{(1)}$

\section{CONCLUSION}

The addition of metformin to neoadjuvant chemotherapy has a nearby significant impact on pathological complete response (PCR) in female patients with advanced breast cancer with no significant increased toxicity.

\section{REFERENCES}

1. Buzatto I, Ribeiro-Silva A, Andrade $J$ et al. (2017): Neoadjuvant chemotherapy with trastuzumab in HER2positive breast cancer: pathologic complete response rate, predictive and prognostic factors. Braz J Med Biol Res., 50 (2): e5674.

2. Cantoria M, Patel H, Boros LG et al. (2014): Metformin and Pancreatic Cancer Metabolism. Intech Open, 7: 159-187.

3. Cortazar P, Geyer CE (2015): Pathological complete response in neoadjuvant treatment of breast cancer. Ann Surg Oncol., 22: 1441-1446.

4. Dai X, Xiang L, Li T et al. (2016): Cancer Hallmarks, Biomarkers and Breast Cancer Molecular Subtypes. J Cancer, 7 (10): 281-1294.

5. De Lorenzi $F$, Loschi $P$, Bagnardi $V$ et al. (2016): Oncoplastic breast-conserving surgery for tumors larger than 2 centimeters: Is it oncologically safe? A matchedcohort analysis. Ann Surg Oncol., 23: 1852-1859.

6. Eisenhauer E, Therasseb P, Bogaertsc J et al. (2009): New response evaluation criteria in solid tumours: Revised RECIST guideline (version 1.1). European Journal of Cancer, 45: 228 -247.

7. Goodwin P (2008): Insulin in the adjuvant breast cancer setting: A novel therapeutic target for lifestyle and pharmacologic interventions? J Clin Oncol., 26: 833-834.

8. Jiralerspong S, Palla S, Giordano $\mathrm{S}$ et al. (2009): Metformin and pathologic complete responses to neoadjuvant chemotherapy in diabetic patients with breast cancer. J Clin Oncol., 27: 3297-3302.

9. Kasznicki J, Sliwinska A, Drzewoski J (2014): Metformin in cancer prevention and therapy. Ann Transl Med., 2 (6): 57.

10. Martin-Castillo B, Pernas S, Dorca J et al. (2018): A phase 2 trial of neoadjuvant metformin in combination with trastuzumab and chemotherapy in women with early HER2-positive breast cancer: the METTEN study. Oncotarget, 9 (86): 35687-35704.

11. Peng M, Darko K, Tao T et al. (2017): Combination of metfomin with chemotherapeutic drugs via different molecular mechanisms. Cancer Treat Rev., 54: 24-33.

12. Pierotti M, Berrino F, Gariboldi $M$ et al. (2013): Targeting metabolism for cancer treatment and prevention: metformin, an old drug with multi-faceted effects. Oncogene, 32 (12): 1475-87.

13. Prowell TM, Pazdur R (2012): Perspective: pathological complete response and accelerated drug approval in early breast cancer. N Engl J Med., 366: 2438-41.

14. Ramos-Esquivel A, Hernández-Steller H et al. (2018): Cyclin-dependent kinase 4/6 inhibitors as first-line treatment for post-menopausal metastatic hormone receptor-positive breast cancer patients: a systematic review and meta-analysis of phase III randomized clinical trials. Breast Cancer, 25 (4): 479-488.

15. Van der Laata A, Ramos-Esquivelb A, Landaverde D (2018): Neoadjuvant Metformin Added to Systemic Therapy Increases Pathological Complete Response in Breast Cancer: A Cross-sectional Study, Mexico Hospital, Costa Rica. Arch Breast Cancer, 5 (1): 32-37. 